Open Access

\title{
Comparing validity of risk measures on newsvendor models in open innovation perspective
}

\author{
Sungyong Choi ${ }^{1}$, KyungBae Park ${ }^{2^{*}}$ (D) and Sang-Oh Shim ${ }^{3}$
}

\author{
* Correspondence: \\ kbpark@sangji.ac.kr \\ ${ }^{2}$ Department of Business \\ Administration, Sangji University, \\ Wonju 26339, Republic of Korea \\ Full list of author information is \\ available at the end of the article
}

\begin{abstract}
In the era of Industry 4.0, firms are facing with greater uncertainty. Accordingly, it is important to select quality risk measures to analyze newsvendor problems under risk. Then, open innovation can be a good remedial option for such risk-averse newsvendors because open innovation can offset the profit losses from risk aversion by sharing revenues in supply chains. To find such risk measures in newsvendor problems, we review various risk measures of risk-averse inventory models and existing articles in inventory management literature. Then we provide a logical reasoning and axiomatic framework to evaluate validity of each risk measure in newsvendor problems - consistency to the four axioms in coherent risk measures. In this framework, the underlying assumptions and managerial insights to the newsvendor problems are examined for each risk measure. Consequently, exponential utility function and coherent measures of risk are selected as two plausible risk measures to analyze multi-product risk-averse newsvendor models.
\end{abstract}

\section{Introduction}

In the era of Industry 4.0, firms are facing with greater uncertainty. Accordingly, we cannot always expect that similar outcomes may be repeated in random situations. The first few outcomes may turn out to be very bad such that they might be unacceptable losses. Then, open innovation can be a good remedial option for such risk-averse newsvendors because open innovation can offset the profit losses from risk aversion by sharing revenues in supply chains (refer to Yoon and Jeong (2017)).

In the literature of inventory management, the (single- or multi-product) newsvendor model, initiated by Arrow et al. (1951), is a well-known classical stochastic inventory replenishment problem in supply chain management literature. In this model, there may exist perishable products with random demand in a single-selling season. Then a newsvendor should decide his optimal ordering quantity for each product in this singleperiod model before demand realization. Because the product demand is only given as a probability distribution, the objective function is represented as a random outcome. If the newsvendor orders too much for any product, all the leftover items are sold at a discounted price; if the newsvendor orders too little, it will lose sales opportunity.

The original model by Arrow et al. (1951) maximizes the expected value of profits without resource constraints and demand substitution. Then the multi-product model

(C) The Author(s). 2017 Open Access This article is distributed under the terms of the Creative Commons Attribution 4.0 International License (http://creativecommons.org/licenses/by/4.0/), which permits unrestricted use, distribution, and reproduction in any medium, provided you give appropriate credit to the original author(s) and the source, provide a link to the Creative Commons license, and indicate if changes were made. 
is decomposable into multiple single-product models in each product and has a simple analytical closed-form optimal solution for each product. This solution is known as a fractile, described with overage and underage profits, of the arbitrary (cumulative) demand distribution function. Thus, it can characterize the optimal solution effectively with underage and overage profits as well as its solvability as a closed-form solution. Owing to its simple solution with trade-off analysis between underage and overage profits, it has many applications in industries such as overbooking problems or facility capacity problems.

Since Arrow et al. (1951), many variations of multi-product newsvendor models have been studied in literature. Hadley and Whitin (1963) add a resource constraint and suggest solution methods using Lagrangian multipliers. Van Ryzin and Mahajan (1999) study a multi-product newsvendor with demand substitution. In both of Hadley and Whitin (1963) and van Ryzin and Mahajan (1999), multi-product models is not decomposable, so we need to consider all the products simultaneously. In that sense, a multi-product newsvendor model considers heterogeneous expectations in each product at a time and such setting has been found quite common in literature (refer to Lee and Lee (2015)).

Again Arrow et al. (1951) and its variations focus on maximizing the expected (random) profit. That is, the newsvendor selects his optimal solution based on the expected value of the random outcome. Thus, the original model and its variations can be said to be expected-value optimization models and also equivalently risk-neutral models under uncertainty. However, risk neutrality guarantees the best decision only on average. It may be justified by the Law of Large Numbers. However, we cannot expect that the actual single realization is sufficiently close to its expected value. In fact, when the single realization is very much deviated from its expected value, risk-neutral models will lose their validity. Then risk-averse decision making can be a good alternative, instead of risk-neutral decision making.

To overcome drawbacks of risk-neutral models, various risk preferences have been studied in literature. Lee et al. (2016) argues that degree of ambiguity may affect decision makers' risk preferences. More specifically, consumers tend to be more risk-averse with more ambiguous situations and vice versa. In risk-averse models, inventory managers consider the variability of the outcome as well as its expected value. That is, under risk aversion, a risk-averse inventory manager may prefer more stable outcome even if the outcome is worse on average. Schweitzer and Cachon (2000) conducted two empirical experiments to show risk preferences of inventory managers. By the experiments, they showed that inventory managers may be risk-averse for short life-cycle or high-value products. Therefore, risk aversion can capture the decision making of inventory managers at a different angle from risk neutrality and both of them are consistent with rational decision makers. Because risk aversion significantly affects the optimal choices of inventory managers, it is a very interesting and important factor to analyze the optimal choices of inventory managers. In particular, risk aversion has a very good fit to conservative decision makers. Some good industrial examples are energy, environment and sustainability where risk measurement is very important.

This paper aims to extend the series of previous works, Choi and Ruszczyński (2008), Choi et al., 2011and Choi and Ruszczyński (2011). In those three papers, they conducted the extensive literature review for various risk measures used in the inventory 
management literature and categorized the risk measures into four typical approaches. Then they selected coherent measures of risk as quality risk measures in Choi and Ruszczyński (2008) and Choi et al. (2011) and an exponential utility function in Choi and Ruszczyński (2011), respectively. In each paper, a logical justification was given for using such specific risk measure selected. Then the optimal policy of the newsvendor models was studied by providing several analytical propositions and numerical insights. On the other hand, we examine such logical justifications in those papers more comprehensively and deeply. As a result, we provide a logical reasoning and then axiomatic framework to compare the validity of such risk measures in multi-product newsvendor models by analyzing the underlying assumptions and managerial insights.

In order to find plausible risk models in newsvendor problems, we focus on the measures based on risk aversion. Then we consider risk neutrality for a reference purpose only. For this purpose, the well-known Prospect Theory and loss aversion, initiated by Kahneman and Tverski (1979), are not considered in this paper. The Prospect Theory assumes that people are risk-averse for their gains, but risk-seeking for their losses. It can explain why sometimes people may buy lottery and insurance together, which was not explained by expected utility theory. This situation may be consistent to individual decision makers, but not inventory managers in a company because inventory managers do not have to carry the products incurring losses. Loss aversion is a concept introduced first by Kahneman and Tverski (1979). It refers to the tendency of an individual decision maker who prefers avoiding losses to obtaining gains. However, in a successive work in the Prospect Theory, Tverski and Kahneman, 1992revealed that loss aversion does not occur in routine transactions (refer to Novemsky and Kahneman (2005)), which describe typical inventory decision-making situations.

The remainder of this paper is organized as follows: First, we briefly review the four typical approaches in $\mathbb{1}$. Second, we conduct a literature review in risk-averse inventory models in $\mathbb{} 2$. Third, we discuss the validity of risk measures for newsvendor problems in $\$ 3$. Forth, we show newsvendor problem formulations in $\$ 4$. Lastly, we conclude this paper by summarizing the main results and suggesting some extensions of the paper in $\$ 5$.

\section{Risk measures}

Due to the aforementioned reasons in $\$ 1$, risk-averse newsvendor models have been recently studied very actively with various risk measures in inventory management literature. Choi et al. (2011) had an attempt to categorize the risk measures of risk-averse inventory models in inventory Management literature. Then the authors summarize the typical approaches of risk measures into four groups. They are expected utility theory, stochastic dominance, chance constraints and mean-risk analysis. Although these four categories of risk measures are different from each other, they are closely related and consistent to some extent. In this paper, we continue to use this four-group classification in Choi et al. (2011).

\section{Expected utility theory}

In the utility function approach, inventory managers optimize the expected value of their utility function, instead of the expected outcomes. Then the optimization model of utility function approach can be represented as follows: 
Consider an optimization model where the decision vector $x$ affects a random performance measure, $\phi_{x}$. Here, for all $x \in \boldsymbol{N}$ with $\boldsymbol{N}$ being a vector space, $\phi_{x}: \Omega \rightarrow \mathbb{R}$ is a measurable function on a probability space $(\Omega, \mathcal{F}, P)$ where $\Omega$ is the sample space, $\mathcal{F}$ is a $\sigma$-algebra on $\Omega$ and $P$ is a probability measure on $\Omega$. Then, the modern theory of the expected utility by von.

von Neumann and Morgenstern (1944) derives, from simple axioms, the existence of a nondecreasing utility function, which transforms (in a nonlinear way) the observed outcomes. That is, each rational decision maker has a nondecreasing utility function $u(\cdot)$ such that he prefers random outcome $\phi_{1}$ over $\phi_{2}$ if and only if $\left[u\left(\phi_{1}\right)\right]>\mathbb{E}\left[u\left(\phi_{2}\right)\right]$, and then he optimizes, instead of the expected outcome, the expected value of the utility function. Therefore, the decision maker solves the following optimization model.

$$
\max \mathbb{E}\left[u\left(\phi_{x}\right)\right], \text { for } x \in X
$$

where $\phi_{x}$ is an (measurable) outcome function. From now on, $\phi_{x}$ denotes a profit function in this paper. When the performance measure is defined as a profit function, a risk-averse decision maker is consistent to the second-order stochastic dominance and he has a concave and nondecreasing utility function. Since Eeckhoudt et al. (1995), an approach of utility functions has been popular in risk-averse newsvendor models. In Eeckhoudt et al. (1995), nondecreasing and concave utility function are used to analyze risk-averse newsvendor models.

In this paper, we select an exponential utility function among various nondecreasing and concave utility functions. Choi and Ruszczyński (2011) point out that.

Exponential utility function is a particular form of a nondecreasing and concave utility function. It is also the unique function to satisfy constant absolute risk aversion (CARA) property. For those reasons, exponential utility function has been used frequently in finance and also in the supply chain management literature such as Bouakiz and Sobel (1992) and Chen et al. (2007).

\section{Stochastic dominance}

Stochastic dominance is the sequence of the partial orders defined on the space of random variables in a nested way such as the first-order, the second-order, the higherorders than the second and so on. This sequence of relations allow pairwise comparison of different random variables (see Lehmann (1955) and Hadar and Russell (1969)) and lower-orders are stronger relations in the sequence. In the sequence of the relations, the second-order stochastic dominance is consistent to risk aversion.

Then an important property of stochastic dominance relations is its consistency to utility functions. That is, a random variable $\phi_{1}$ dominates $\phi_{2}$ by a stochastic dominance relation is equivalent that the expected utility of $\phi_{1}$ is better than that of $\phi_{2}$ for all utility functions in a certain family of utility functions. For the first- and second-order stochastic dominance relations, this property is represented as follows:

$\phi_{1} \succcurlyeq_{(1)} \phi_{2} \Leftrightarrow \mathbb{E}\left[u\left(\phi_{1}\right)\right] \geq \mathbb{E}\left[u\left(\phi_{2}\right)\right]$, for every nondecreasing $U[\cdot]$.

$\phi_{1} \succcurlyeq_{(2)} \phi_{2} \Leftrightarrow \mathbb{E}\left[u\left(\phi_{1}\right)\right] \geq \mathbb{E}\left[u\left(\phi_{2}\right)\right]$, for every nondecreasing and concave $U[\cdot]$

In spite of such nice properties, stochastic dominance does not have a simple computational method unfortunately for its implementation by itself. Thus, it has been mainly used as a reference criterion to evaluate the legitimacy of risk-averse inventory models. 


\section{Chance constraints}

Chance constraints add some constraints on the probabilities that measure the risk such as:

$$
P\left(\phi_{x} \geq \eta\right) \geq 1-\alpha
$$

where $\eta$ is a fixed target value and $\alpha \in(0,1)$ is the maximum level of risk of violating the stochastic constraint, $\phi_{x} \geq \eta$. Then, we consider the following optimization model.

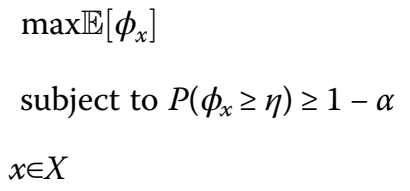

In finance, chance constraints are very popular as the name of VaR (Value-at-Risk). For consistency to stochastic dominance, $\mathrm{VaR}$ is a relaxed version of the first-order stochastic dominance, but might violate the second-order stochastic dominance.

\section{Mean-risk analysis}

Mean-risk analysis provides efficient solutions and quantifies the problem in a clear form of two criteria: the mean (the expected value of the outcome) and the risk (a scalar measured variability of the outcome). In mean-risk analysis, one uses a specified functional $r: \boldsymbol{N} \rightarrow \mathbb{R}$, where $\boldsymbol{N}$ is a certain space of measurable functions on a probability space $(\Omega, \mathcal{F}, P)$ to represent variability of the random outcomes, and then solves the problem:

$$
\min \left\{-\mathbb{E}\left[\phi_{x}\right]+\lambda r\left[\phi_{x}\right]\right\}, \text { for } x \in X \text { where } \lambda \in \mathbb{R}^{+} \cup\{0\}
$$

Here, $\lambda$ is a nonnegative trade-off constant between the expected outcome and the scalar-measured value of the variability of the outcome. This allows a simple trade-off analysis analytically and geometrically.

In the minimization context, one selects from the universe of all possible solutions those that are efficient: for a given value of the mean he minimizes the risk, or equivalently, for a given value of risk he maximizes the mean. Such an approach has many advantages: it allows one to formulate the problem as a parametric optimization problem, and it facilitates the trade-off analysis between mean and risk. However, for some popular dispersion statistics used as risk measures, the mean-risk analysis may lead to inferior conclusion. Thus, it is of primary importance to decide a good risk measure for each type of the decision models to be considered. The two important examples are mean-variance (or mean-standard deviation) model and coherent risk measures.

Mean-variance model

Since the seminal work of Markowitz (1952), mean-variance model has been actively used in the literature and it used the variance of the return as the risk functional, i.e.

$$
r\left[\phi_{x}\right]=\operatorname{Var}\left[\phi_{x}\right]=\mathbb{E}\left[\left(\phi_{x}-\mathbb{E}\left[\phi_{x}\right]\right)^{2}\right]
$$

Since its introduction, many authors have pointed out that the mean-variance model is, in general, not consistent with stochastic dominance rules. It may lead to an optimal solution which is stochastically dominated by another solution. Thus, to overcome drawbacks of mean-variance model, the general theory of coherent measures of risk was 
initiated by Artzner et al. (1999) and extended to general probability spaces by Delbaen (2002).

\section{Coherent measures of risk}

Coherent measures of risk are extensions of mean-risk model to put different variability measures $r[\cdot]$ (e.g. deviation from quantile or semideviation) instead of variance. A formal definition of the coherent measures of risk is presented by following the abstract approach of Ruszczyński and Shapiro (2005 and 2006a).

Let $(\Omega, \mathcal{F})$ be a certain measurable space. A uncertain outcome is represented by a measurable function $\phi_{x}: \Omega \rightarrow \mathbb{R}$. We specify the vector space $\mathcal{Z}$ of the possible functions of $\phi_{x}$; in this case it is sufficient to consider $\mathcal{Z}=\mathcal{L}_{\infty}(\Omega, \mathcal{F}, P)$.

A coherent measure of risk is a functional $\rho: \mathcal{Z} \rightarrow \mathbb{R}$ satisfying the following axioms:

Convexity: $\rho\left(\alpha \phi_{1}+(1-\alpha) \phi_{2}\right) \leq \alpha \rho\left(\phi_{1}\right)+(1-\alpha) \rho\left(\phi_{2}\right)$, for all $\phi_{1}, \phi_{2} \in \mathcal{Z}$ and all $\alpha \in[0,1]$;

Monotonicity: If $\phi_{1}, \phi_{2} \in \mathcal{Z}$ and $\phi_{1} \geqslant \phi_{2}$, then $\rho\left(\phi_{1}\right) \leq \rho\left(\phi_{2}\right)$;

Translation Equivariance: If $\mathrm{a} \in \mathbb{R}$ and $\phi_{1} \in \mathcal{Z}$, then $\rho\left(\phi_{1}+a\right)=\rho\left(\phi_{1}\right)-a$;

Positive Homogeneity: If $\mathrm{t} \geq 0$ and $\phi_{1} \in \mathcal{Z}$, then $\rho\left(t \phi_{1}\right)=t \rho\left(\phi_{1}\right)$.

An optional axiom in coherent measures of risk is law-invariance. A coherent measure of risk $\rho(\cdot)$ is called law-invariant, if the value of $\rho\left(\phi_{1}\right)$ depends only on the distribution of $\phi_{1}$, that is $\rho\left(\phi_{1}\right)=\rho\left(\phi_{2}\right)$ if $\phi_{1}$ and $\phi_{2}$ have identical distributions. Acerbi (2004) summarizes the meaning of this property that a law-invariant coherent measure of risk gives the same risk for empirically exchangeable random outcomes. Law-invariance looks so obvious that it is no wonder even if most risk practitioners take it for granted. However, it also implies that, for a coherent measure of risk $\rho$ which is not lawinvariant, $\rho\left(\phi_{1}\right)$ and $\rho\left(\phi_{2}\right)$ may be different even if $\phi_{1}$ and $\phi_{2}$ have same probability distribution. This apparent paradox can be resolved by reminding the formal definition of random variables. Actually, one needs to determine simultaneously "probability law" and "field of events" endowed with a $\sigma$-algebra structure to define a random variable. Thus, the two random variables with same probability distributions can be different and may have different values of $\rho$. An example of the coherent measure of risk which is not law-invariant is the so-called worst conditional expectation $W C E_{\alpha}$ defined in Artzner et al. (1999).

$$
W C E_{\alpha}=-\inf \left\{\mathbb{E}\left[\phi_{1} \mid A\right]: A \in \mathcal{A}, P[A]>\alpha\right\}
$$

The infimum of conditional expectations $\mathbb{E}\left[\phi_{1} \mid A\right]$ is taken on all the events $A$ with probability larger than $\alpha$ in the $\sigma$-algebra $\mathcal{A}$. However, under certain conditions on nonatomic probability space, this risk measure becomes law-invariant and coincides with a famous risk measure CVaR (Conditional Value-at-Risk). For more technical details, see Acerbi and Tasche (2002), Delbaen (2002) and Kusuoka (2003).

Because coherent measures of risk are any functionals to satisfy the four axioms above, their functional forms are not determined uniquely. The two popular examples are obtained to put deviation-from-quantile, $r_{\beta}[\cdot]$ with $\lambda \in[0,1]$, or semideviation of order $k \geq 1, \sigma_{k}[\cdot]$ with $\lambda \in[0,1 / \beta]$, into $r[\cdot]$, variability of the outcome:

$$
\sigma_{k}\left[\phi_{1}\right]=\mathbb{E}\left[\left\{\left(\mathbb{E}\left[\phi_{1}\right]-\phi_{1}\right)^{+}\right\}^{k}\right]^{\frac{1}{k}}
$$




$$
r_{\beta}\left[\phi_{1}\right]=\min \mathbb{E}\left[\max \left((1-\beta)\left(\eta-\phi_{1}\right), \beta\left(\phi_{1}-\eta\right)\right)\right], \text { for } \eta \in \mathbb{R} \text { with } \beta \in(0,1)
$$

The optimal $\eta$ in the eq. (5) is the $\beta$-quantile of $\phi_{1}$. Then CVaR is a special case of mean-deviation-from-quantile when $\lambda=1 / \beta$. All these results can be found at Ruszczyński and Shapiro (2006a) and Choi (2009) with a sign adjustment.

\section{Literature review}

Choi et al. (2011) also provided a comprehensive literature review in risk-averse inventory models since the seminal works of Lau (1980) and Eeckhoudt et al. (1995). In this paper, we provide a summary of literature of the risk-averse newsvendor models studied after Choi et al. (2011) at Table 1 where we classify and tabulate the literature by model types (as columns) and risk measures used (as rows). The key research question from the literature is the impact of degree of risk aversion to the optimal ordering quantity with parametric and comparative static analysis. A common finding from literature is that higher degree of risk aversion results in fewer optimal ordering quantities because higher ordering quantity implies higher variability of the profits. Then, risk-averse newsvendor tends to decrease ordering quantity to avoid higher risk.

Yang et al. (2008) consider a single-product risk-averse newsvendor with a capacity constraint for ordering quantity. They select two risk measures, CVaR (Conditional Value-at-Risk) and VaR (Value-at-Risk), for their models. As a result, they provide closed-form optimal solution with both risk measures and confirm their results with numerical examples. Chen et al. (2009) study a single-product newsvendor of stochastic price-dependent demand with CVaR. That is, their models are joint models of ordering quantity and price. The key research questions are to characterize the optimal order quantity and prices and to conduct comparative statics analysis with respect to model parameters for additive and multiplicative demand cases. In addition, they compare their results with those in the corresponding risk-neutral models of stochastic pricedependent demand. Özler et al. (2009) consider a multi-product newsvendor with a Value-at-Risk constraint. They also consider a single-product newsvendor as a special case. For a single-product system, they obtain the closed-form optimal ordering quantity which is the same result of Gan et al. (2004). Their biggest contribution to the literature is that for a two-product system, they obtain the mathematical formulation of

Table 1 Summary of the literature on risk-averse newsvendor models

\begin{tabular}{|c|c|c|c|}
\hline \multicolumn{2}{|l|}{ Risk Measures $\backslash$ Model Types } & $\begin{array}{l}\text { Single-product } \\
\text { Newsvendor }\end{array}$ & $\begin{array}{l}\text { Multi-product } \\
\text { Newsvendor }\end{array}$ \\
\hline \multicolumn{2}{|l|}{ Utility Function } & $\begin{array}{l}\text { Choi and Ruszczyński } \\
\text { (2011) }\end{array}$ & $\begin{array}{l}\text { Choi and Ruszczyński } \\
\text { (2011) }\end{array}$ \\
\hline \multicolumn{2}{|l|}{ Stochastic Dominance } & \multicolumn{2}{|c|}{$\begin{array}{l}\text { It is a reference criterion. } \\
\text { Thus, it is not directly applicable for } \\
\text { implementation. }\end{array}$} \\
\hline \multicolumn{2}{|c|}{ Chance Constraints (or Value-at-risk) } & Özler et al. (2009) & Özler et al. (2009) \\
\hline \multirow[t]{4}{*}{$\begin{array}{l}\text { Mean-risk Coherent } \\
\text { Analysis }\end{array}$} & $\begin{array}{l}\text { Mean-deviation from quantile } \\
\text { or mean-semideviation }\end{array}$ & $\begin{array}{l}\text { Chen et al. (2009) } \\
\text { Yang et al. (2008) }\end{array}$ & N/A \\
\hline & General Coherent Risk Measures & $\begin{array}{l}\text { Choi and Ruszczyński } \\
\text { (2008) }\end{array}$ & $\begin{array}{l}\text { Choi and Ruszczyński } \\
\text { (2011) }\end{array}$ \\
\hline & Mean-variance & N/A & N/A \\
\hline & Mean-standard deviation & $\mathrm{N} / \mathrm{A}$ & N/A \\
\hline
\end{tabular}


mixed integer programming where the objective function is nonlinear and the constraints are mixed linear and nonlinear functions. Then, they conducted their numerical analysis to confirm their analytical results under multi-variate exponential demands.

\section{Discussion of validity of risk measures in newsvendor models}

\section{Four axioms in coherent measures of risk}

The four axioms (Convexity, Monotonicity, Translation Equivariance and Positive Homogeneity) in coherent measures of risk have attractive features and implications to analyze newsvendor problems and thus the axioms make coherent measures of risk worth considering. Although these four axioms are briefly discussed at the previous studies in literature, none of them had an attempt to consider all of the four axioms in a comparative sense. More specifically, based on the four axioms, we develop them as an axiomatic framework to analyze the validity of newsvendor problems. The optional axiom, LawInvariance, does not have a practical meaning, so we do not consider it in this section.

- Convexity axiom means that the global risk of a portfolio should be equal or less than the convex combination of its partial risks. Because lower measured risk is better in coherent measures of risk, this axiom is consistent with the diversification effects.

- In the Monotonicity axiom, $\phi_{1} \geqslant \phi_{2}$ means that $\phi_{1}$ is always preferred to $\phi_{2}$ for all possible scenarios. Thus, this axiom means that if portfolio 1 always has better values than portfolio 2 under all possible scenarios, then the measured risk of the portfolio 1 should be less than the measured risk of portfolio 2. By satisfying this axiom, coherent measures of risk are consistent with the second-order stochastic dominance.

- Translation Equivariance axiom means that the existence of a constant cost (or gain) is equivalent to equally decreasing (or increasing) the vendor's performance measure. Thus, fixed parts can be separated equivalently from the vendor's random performance measure at every possible state of nature. Thus, this axiom allows one to draw a comparison between the only random parts of different random performance measures and thus rank risk properly (see Artzner et al. (1999)). However, this axiom is contradictory to initial endowment effects (refer to Choi et al. (2011)).

- Positive Homogeneity axiom guarantees that the optimal solution is invariant to rescaling of units such as currency (e.g., from dollars to pounds) or considering the total profit or the average profit per product. In addition, this axiom guarantees no diversification effects in a limiting case when the multivariate demand has a perfect positive correlation (see Choi et al. (2011)).

These features are derived regardless of any specific problem formulations in multiproduct newsvendor problems. That is, these features and implications can be directly applied in any type newsvendor problems with different formulations to evaluate the validity of risk measures.

\section{The axiomatic framework}

In this subsection, we compare the validity of various risk measures in newsvendor problems by our axiomatic framework based on the four axioms of coherent risk 
measures. The axiomatic approach provides a clear standard to evaluate risk measures in risk-averse newsvendor models. (Table 2).

Stochastic dominance is a reference criterion to give pairwise comparison between different random outcome. Thus, it is not directly implemented for its application.

Chance constraints have been actively used in finance historically. In financial terms, they are intuitive and easy to understand. However, they generally violate Convexity, which implies that chance constraints may penalize diversification instead of encouraging it. Historically, the Convexity has been a controversial axiom in finance literature due to the popularity of $\mathrm{VaR}$ in financial markets. However, such situations may be justified in finance literature such as insurance industry, but very different from that in newsvendor problems. In fact, the Convexity axiom is especially valid in newsvendor models. Each product is very likely to have some nonzero value in newsvendor models because very small amounts will be sold almost always for each product (refer to Choi et al. (2011) and Choi and Ruszczyński (2011)).

Mean-variance and mean-standard deviation model have been very well-known since the seminal work of Markowitz (1952). The mean-variance model satisfies the Translation Equivariance axiom only. Mean-standard deviation model satisfies additionally Positive Homogeneity as well as Translation Equivariance, but not Convexity and Monotonicity.

Since its introduction, many authors have pointed out that the mean-variance and mean-standard deviation models are, in general, not consistent with stochastic dominance rules, nor the Monotonicity axiom. Because both models consider overperformance and under-performance equally, they are not so-called downside risk measures and may lead to an optimal solution which is stochastically dominated by another solution. Thus, to overcome drawbacks of mean-variance model, the general theory of coherent measures of risk was initiated by Artzner et al. (1999) and extended. We provide a specific and simple counterexample that a mean-variance model violate the monotonicity axiom in Table 3.

In Table 3, we set up $\Omega=\left\{\omega_{1}, \omega_{2}\right\}$ and $P\left(\omega_{1}\right)=P\left(\omega_{2}\right)=0.5$. Then larger value is always preferred to smaller value in this table. Each random variable $\phi_{1}$ and $\phi_{2}$ has a value for any possible states of nature, $\omega_{1}$ and $\omega_{2}$, and $\phi_{1}(\omega)$ is always better than $\phi_{2}(\omega)$ for all $\omega \in \Omega$. Thus, $\phi_{1}$ dominates $\phi_{2}$ by the rule of statewise dominance and this table is a

Table 2 Comparison between risk measures in newsvendor problems

\begin{tabular}{|c|c|c|c|c|c|c|}
\hline \multirow{2}{*}{\multicolumn{3}{|c|}{ Risk measures $\backslash$ Model types }} & \multicolumn{4}{|c|}{$\begin{array}{l}\text { Consistency to the four axioms for coherent risk } \\
\text { measures }\end{array}$} \\
\hline & & & Convexity & Monotonicity & $\begin{array}{l}\text { Translation } \\
\text { Equivariance }\end{array}$ & $\begin{array}{l}\text { Positive } \\
\text { Homogeneity }\end{array}$ \\
\hline \multicolumn{3}{|c|}{ Utility Function } & Yes & Yes & No & No \\
\hline \multicolumn{3}{|c|}{ Stochastic Dominance } & \multicolumn{4}{|c|}{$\begin{array}{l}\text { It is a reference criterion. } \\
\text { Thus, it is not directly applicable for implementation. }\end{array}$} \\
\hline \multicolumn{3}{|c|}{ Chance Constraints (or Value-at-risk) } & No & Yes & Yes & Yes \\
\hline \multirow[t]{4}{*}{$\begin{array}{l}\text { Mean-risk } \\
\text { Analysis }\end{array}$} & \multirow[t]{2}{*}{ Coherent } & $\begin{array}{l}\text { Mean-deviation from quantile or } \\
\text { mean-semideviation }\end{array}$ & \multicolumn{4}{|c|}{ Yes for all of the four axioms } \\
\hline & & General Coherent Risk Measures & \multicolumn{4}{|c|}{ Yes for all of the four axioms } \\
\hline & \multirow{2}{*}{$\begin{array}{l}\text { Non- } \\
\text { Coherent }\end{array}$} & Mean-variance & No & No & Yes & No \\
\hline & & Mean-standard deviation & No & No & Yes & Yes \\
\hline
\end{tabular}


Table 3 A counterexample to show imperfection of mean-variance model

\begin{tabular}{lll}
\hline & $\omega_{1}$ & $\omega_{2}$ \\
\hline$\varphi_{1}$ & -1 & 3 \\
$\varphi_{2}$ & -1 & -1 \\
\hline
\end{tabular}

good example where an efficient solution (in the sense from mean-risk analysis) is dominated by another solution. Clearly, $\phi_{1}$ may be preferred to $\phi_{2}$. However, $\mathbb{E}\left(\phi_{2}\right)-1$. $\operatorname{Var}\left(\phi_{2}\right)=-1>-3=\mathbb{E}\left(\phi_{1}\right)-1 \cdot \operatorname{Var}\left(\phi_{1}\right)$. This implies that $\phi_{2}$ is more preferable to $\phi_{1}$ under mean-variance criterion, which is inconsistent with the Monotonicity axiom.

\section{Selection of risk measures in newsvendor problems}

In summary, expected utility theory and coherent risk measures share the Convexity and Monotonicity axioms when a newsvendor has a nondecreasing and concave function. However, expected utility theory does not satisfy the Translation Equivariance and Positive Homogeneity. General coherent measures of risk are consistent to the firstand second-order stochastic dominance relations and satisfy all the four axioms. Thus, Translation Equivariance and Positive Homogeneity axioms are crucial to decide which one is better to use between utility function approach and coherent measures of risk.

The initial endowment effects, firstly theorized by Thaler (1980) in behavioral economics, mean that the initial states of the variables may affect the optimal decision. Sometimes the effects may have a significant role for inventory managers. Therefore, if a newsvendor takes initial endowment effects strongly, then coherent measures of risk may not be preferred by this newsvendor. Such effects can be captured by utility function approach, but not by coherent measures of risk. Thus, if newsvendors show initial endowment effects significantly, utility function approach is better to use to analyze the newsvendor problems. More specifically, exponential utility function is a particular form of a nondecreasing and concave utility function. It is also the unique function to satisfy constant absolute risk aversion property. For those reasons, exponential utility function has been used frequently in finance and also in supply chain management literature such as Bouakiz and Sobel (1992), Chen et al. (2007) and Choi and Ruszczyński (2011). However, the existence of initial endowment effects is still controversial (see Hanemann (1991) and Shogren et al. (1994)).

On the other hand, Positive Homogeneity implies invariance of the optimal solution from denomination of the currency to guarantee consistence to rational risk-averse decision making. Choi et al. (2011) provide a numerical example where they compare solutions of a single-product newsvendor model with coherent measures of risk, exponential utility function and mean-variance. They initially select parameters in each risk measure so that they have the same optimal solution when the unit of profit is measured as one dollar. Then they change the unit of profit continuously by denomination. Then the optimal solution with coherent measures of risk is unchanged, but the solutions significantly change with the other risk measures. Because utility functions are not compatible with Positive Homogeneity, they also have some drawbacks to analyze newsvendor problems.

In conclusion, considering relative advantages and disadvantages of using each type risk measure, exponential utility function approach and coherent measures of risk are 
two plausible risk measures to analyze newsvendor model by the consideration with the axiomatic approach.

\section{Conclusion}

In this paper, we have examined various risk measures in newsvendor problems. By focusing on the four axioms of coherent risk measures, we have compared the four typical approaches; expected utility theory, stochastic dominance, chance constraints and mean-risk analysis. As a result, an exponential utility function and coherent risk measures are selected as two quality risk measures for newsvendor problems.

It is natural that the newsvendors are risk-averse when they are in a preliminary transition stage to open innovation. Then, in order to handle risk aversion properly, it is necessary to find a quality risk measures for such cases. Due to this reason, we consider the validity of risk measures for the risk-averse newsvendor models when their levels of open innovation are relatively low. Finally, our contributions to literature are can be summarized as follows: First, we conduct an extensive and rigorous literature review in risk measures and newsvendor problems in a perspective of open innovation. Second, we discuss the relationship between two conflicting risk preferences, risk aversion and neutrality, with open innovation. Last, we provide an axiomatic framework to verify the validity of various risk measures used in real world as well as the literature of this research stream.

We believe that there is an important extension that can be addressed in this axiomatic framework. In this paper, we discuss meaning and implications of the four axioms in coherent risk measures in newsvendor models. For a multi-period case, dynamic version of coherent risk measures were also analyzed in the literature (refer to Riedel (2004), Kusuoka and Morimoto (2004), Cheridito et al. (2006) and Ruszczyński and Shapiro (2006b)). Then, with appropriate adjustments, this axiomatic approach can be a good starting point of constructing another axiomatic framework to compare the validity of various risk measures for a multi-period case.

Acknowledgements

Not applicable

Funding

Not applicable

Availability of data and materials

The dataset(s) supporting the conclusions of this article is (are) included within the article.

Authors' contributions

SC contributed to the overall idea, theoretical development, and writing of the manuscript; KBP contributed to discussions of risk measures on open innovation, as well as the preparation and publishing of the paper; S-OS contributed to detailed writing and discussions of risk measures on sustainability. All authors read and approved the final manuscript.

Competing interests

The authors declare that they have no competing interests.

\section{Publisher's Note}

Springer Nature remains neutral with regard to jurisdictional claims in published maps and institutional affiliations.

Author details

${ }^{1}$ Division of Business Administration, Yonsei University, Wonju 26493, Republic of Korea. ${ }^{2}$ Department of Business Administration, Sangji University, Wonju 26339, Republic of Korea. ${ }^{3}$ Department of Business Administration and Accounting, Hanbat National University, Daejeon 34158, Republic of Korea. 
Received: 30 September 2017 Accepted: 30 November 2017

Published online: 03 January 2018

\section{References}

Acerbi, C. (2004). Coherent representation of subjective risk aversion. In G. Szegö (Ed.), Risk measures for the 21st century (pp. 147-207). Chichester: Wiley.

Acerbi, C., \& Tasche, D. (2002). On the coherence of expected shortfall. Journal of Banking and Finance, 26(7), 1487-1503.

Arrow, K., Harris, T., \& Marschak, J. (1951). Optimal inventory policy. Econometrica, 19(3), 250-272.

Artzner, P., Delbaen, F., Eber, J., \& Heath, D. (1999). Coherent measures of risk. Mathematical Finance, 9(3), $203-228$.

Bouakiz, M., \& Sobel, M. (1992). Inventory control with an exponential utility criterion. Operations Research, 40(3), 603-608.

Chen, X., Sim, M., Simchi-Levi, D., \& Sun, P. (2007). Risk aversion in inventory management. Operations Research, 55, 828-842.

Chen, Y., Xu, M., \& Zhang, Z. (2009). A risk-averse newsvendor model under the CVaR criterion. Operations Research, 57(4), 1040-1044.

Cheridito, P., Delbaen, F., \& Kupper, M. (2006). Dynamic monetary risk measures for bounded discrete-time processes. Electronic Journal of Probability, 11, 57-106.

Choi, S. (2009). The risk-averse newsvendor models, ph.D. Dissertation, Rutgers University.

Choi, S., \& Ruszczyński, A. (2008). A risk-averse newsvendor with law-invariant coherent measures of risk. Operations Research Letters, 36, 77-82.

Choi, S., \& Ruszczyński, A. (2011). A risk-averse newsvendor with exponential utility function. European Journal of Operational Research, 214, 78-84.

Choi, S., Ruszczyński, A., \& Zhao, Y. (2011). A multiproduct risk-averse newsvendor with law-invariant coherent measures of risk. Operations Research, 59(2), 346-364.

Delbaen, F. (2002). Coherent risk measures on general probability space. In K. Sandmann \& P. J. Schönbucher (Eds.), Advances in finance and Stochastics (pp. 1-37). Heidelberg: Springer.

Eeckhoudt, L., Gollier, C., \& Schlesinger, H. (1995). The risk-averse (and prudent) newsboy. Management Science, 41(3), 786-794.

Gan, X., Sethi, S. P., \& Yan, H. (2004). Coordination of supply chains with risk-averse agents. Production and Operations Management, 14(1), 80-89.

Hadar, J., \& Russell, W. (1969). Rules for ordering uncertain prospects. The American Economic Review, 59, $25-34$.

Hadley, G., \& Whitin, T. M. (1963). Analysis of inventory systems. Englewood Cliffs: Prentice-Hall.

Hanemann, W. M. (1991). Willingness to pay and willingness to accept: How much can they differ. The American Economic Review, 81(3), 635-647.

Kahneman, D., \& Tverski, A. (1979). Prospect theory: An analysis of decision under risk. Econometrica, 47, $263-291$.

Kusuoka, S. (2003). On law invariant coherent risk measures. Adv. Math. Econ., 3, 83-95.

Kusuoka, S. \& Morimoto, Y. (2004). Homogeneous law invariant coherent multiperiod value measures and their limits. Working Paper, Graduate School of Mathematical Science, University of Tokyo.

Lau, H. (1980). The newsboy problem under alternative optimization objectives. The Journal of the Operational Research Society, $31(6), 525-535$.

Lee, S., \& Lee, K. (2015). Heterogeneous expectations leading to bubbles and crashes in asset markets: Tipping point, herding behavior and group effect in an agent-based model. Journal of Open Innovation: Technology, Market, and Complexity, 1(11). https://doi.org/10.1186/s40852-015-0014-8.

Lee, S., Workman, J. E., \& Jung, K. (2016). Brand relationships and risk: Influence of risk avoidance and gender on brand consumption. Journal of Open Innovation: Technology, Market, and Complexity, 2(14). doi:10.1186/s40852-016-0041-0.

Lehmann, E. (1955). Ordered families of distributions. Annals of Mathematical Statistics, 26, 399-419.

Markowitz, H. (1952). Portfolio selection. Journal of Finance, 7, 77-91.

Novemsky, N., \& Kahneman, D. (2005). The boundaries of loss aversion. Journal of Marketing Research, 42, 119-128.

Özler, A., Tan, B., \& Karaesmen, F. (2009). Multi-product newsvendor problem with value-at-risk constraints. International Journal of Production Economics, 117, 244-255.

Riedel, F. (2004). Dynamic coherent risk measures. Stochastic Processes and their Applications, 112, 185-200.

Ruszczyński, A., \& Shapiro, A. (2005). Optimization of risk measures. In G. Calafiore \& F. Dabbene (Eds.), Probabilistic and randomized methods for design under uncertainty (pp. 117-158). London: Springer-Verlag.

Ruszczyński, A., \& Shapiro, A. (2006a). Optimization of convex risk functions. Mathematics of Operations Research, 31(3), $433-452$.

Ruszczyński, A., \& Shapiro, A. (2006b). Conditional risk mappings. Mathematics of Operations Research, 31(3), 544-561.

Schweitzer, M., \& Cachon, G. (2000). Decision bias in the newsvendor problem with a known demand distribution: Experimental evidence. Management Science, 46(3), 404-420.

Shogren, J. F., Shin, S., Hayes, D. J., \& Kliebenstein, J. B. (1994). Resolving differences in willingness to pay and willingness to accept. The American Economic Review, 84(1), 255-270.

Thaler, R. (1980). Toward a positive theory of consumer choice. Journal of Economic Behavior and Organization, 1, 39-60.

Tverski, A., \& Kahneman, D. (1992). Advances in prospect theory: Cumulative representation of uncertainty. Journal of Risk and Uncertainty, 5, 297-323.

van Ryzin, G. J., \& Mahajan, S. (1999). On the relationship between inventory cost and variety benefits in retail assortments. Management Science, 45, 1496-1509.

von Neumann, J., \& Morgenstern, O. (1944). Theory of games and economic behavior. Princeton: Princeton University Press.

Yang, L., Wang, M., \& Li, W. (2008). Two types of risk-averse newsvendor models with limited capacity. Systems Engineering - Theory \& Practice, 28(4), 35-42.

Yoon, S., \& Jeong, S. (2017). Effects to implement the open-innovation coordinative strategies between manufacturer and retailer in reverse supply chain. Journal of Open Innovation: Technology, Market, and Complexity, 3(2). doi:10. 1186/s40852-017-0054-3. 